\title{
Incident gout and erectile dysfunction: is hyperuricaemia the elephant in the room?

\author{
Abhishek Abhishek ${ }^{*}$ and Michael Doherty
}

See related research by Sultan et al., https://arthritis-research.biomedcentral.com/articles/10.1186/s13075-017-1322-0

\begin{abstract}
The first prospective population-based study to examine risk of erectile dysfunction in men with gout in the western world has been published. It reports that following their first diagnosis of gout, men have a $31 \%$ higher risk of erectile dysfunction than matched controls, although the absolute increase in risk is small. Of interest, the incidence of erectile dysfunction reported in this study is tenfold higher than those reported in nation-wide cohort studies from Taiwan. There is a need for further prospective cohort studies to examine the possible mechanistic association between gout, hyperuricaemia and erectile dysfunction.
\end{abstract}

Keywords: Gout, Erectile dysfunction

The primary care-based prospective cohort study by Abdul Sultan et al. [1] using data from the UK's Clinical Practice Research Datalink (CPRD) reports that following their first diagnosis of gout, men have a 31\% higher risk of erectile dysfunction (ED) than matched controls, although the absolute increase in risk is small (e.g. 0.6\% for a new consultation for ED, and $0.3 \%$ for receiving a prescription), reflecting a limited attributable effect on ED compared to other factors.

The authors are to be congratulated for undertaking this first prospective population-based study to examine risk of ED in men with gout in the western world. Only three other studies have examined ED in men with gout. One small hospital-based USA study surveyed people attending rheumatology clinics and reported that $76 \%$ of people with gout $(n=81)$ had ED compared to $51 \%$ of non-gout controls $(\mathrm{n}=115) \quad$ [2]. Interestingly, the

* Correspondence: Abhishek.abhishek@nottingham.ac.uk

Academic Rheumatology, Division of Rheumatology, Orthopaedics, and

Dermatology, School of Medicine, University of Nottingham, Nottingham, UK incidence rate of ED reported in this CPRD study is tenfold higher than those reported in two nation-wide cohort studies using the National Health Insurance Research Database (NHIRD) in Taiwan [3, 4]. It is possible that this difference results from cultural reasons or lack of health insurance cover for ED in Taiwan [1]. However, hazard ratios for ED in those with gout in the Taiwan studies were comparable to the current study [1, 3, 4]. Given the prospective study design and source of recruitment we feel that this study provides reasonable estimates of rates of ED in men with gout in the western world [1], though the real prevalence is likely to be higher because many men may not consult their GP about it. Due to data limitations, the authors could not classify ED according to cause. However, data from the NHIRD showed a greater risk of incident organic ED than psychogenic ED in men with gout compared to men without gout (odds ratio (95\% confidence interval) $1.52(1.03-2.22)$ vs. 1.18(1.03-1.35), respectively) [4], which suggests an important role for hyperuricaemia in causing vasculopathy and subsequent ED.

Abdul Sultan et al. [1] performed stratified and multivariate analyses to account for several confounders that may associate with gout and ED, and used landmark analysis to account for survivor bias. Interestingly, they found an elevated risk of ED prior to gout diagnosis, which suggests that ED may result from hyperuricaemia which precedes gout by some years [5]. However, the elevated risk for ED was restricted just to the 1-year period prior to gout diagnosis [1]. This suggests a possible delay between symptom onset and first consultation for gout, or that Read codes for gout are not applied at initial GP consultations, potentially due to diagnostic uncertainty in early stages of illness [6]. However, the control group for the analysis of association between prior diagnosis of ED and subsequent coding for gout was not selected according to the serum uric acid (SUA) and may have undetected hyperuricaemia, thereby minimizing the association. 
Interestingly, a previous CPRD study identified an increased risk of many comorbidities that may relate to chronic hyperuricaemia (including cardiovascular diseases and depression) in up to 10 years prior to initial gout diagnosis and a subsequent increased risk of the same comorbidities in the 5 years following diagnosis [7]. Depression was identified as an association with incident and subsequent gout in the current study and could have contributed to an increase in psychogenic ED. Thus, there is a need for further prospective cohort studies to examine the possible mechanistic association between hyperuricaemia and ED. Given that screening for hyperuricaemia is not standard clinical practice in the UK, such studies cannot be conducted using UK primary care data.

This study found no protective effect of urate lowering treatment (ULT) on the incidence of ED. This is not unexpected as most people with gout in the UK who receive ULT are on suboptimal doses and do not achieve target SUAs of $<6 \mathrm{mg} / \mathrm{dl}$ [8]. Of interest, the authors found a trend towards higher risk of ED in those treated with ULT, although this was not statistically significant. This may be due to residual confounding by indication, as individuals with more severe disease and comorbidities are more likely to receive ULT. Such confounding could have been minimized by propensity score matching or adjustment. Thus, we feel that the lack of risk reduction for incident ED by ULT should not be interpreted as failure of ULT in preventing ED, since the suboptimal dosing of allopurinol in the UK is unlikely to exert any beneficial effect on vascular health, or indeed mortality $[8,9]$.

In summary, health care professionals should be aware that men with gout are at higher risk of ED, and might enquire about this during consultations. Further studies are required to determine whether the association between gout and ED is due to hyperuricaemia, or if gout confers a higher risk of ED than hyperuricaemia alone.

\section{Abbreviations}

CPRD: Clinical Practice Research Datalink; ED: Erectile dysfunction; NHIRD: National Health Insurance Research Database; SUA: Serum uric acid; ULT: Urate lowering treatment

\section{Acknowledgements}

None.

\section{Funding}

None.

Availability of data and materials

Not applicable.

\section{Authors' contributions}

AA and MD wrote the manuscript, corrected it and approved its final version.
Consent for publication

Not applicable.

\section{Competing interests}

$\mathrm{MD}$ and AA report departmental research grants from Astra Zeneca, Oxford Immunotech. MD has received honoraria for ad hoc advisory boards on osteoarthritis or gout from AstraZeneca, Grunenthal, Roche and Mallinckrodt.

\section{Publisher's Note}

Springer Nature remains neutral with regard to jurisdictional claims in published maps and institutional affiliations.

Published online: 10 August 2017

\section{References}

1. Abdul Sultan A, Mallen C, Hayward R, Muller S, Whittle R, Hotston M, Roddy E. Gout and subsequent erectile dysfunction: a population-based cohort study from England. Arthritis Res Ther. 2017;19(1):123.

2. Schlesinger N, Radvanski DC, Cheng JQ, Kostis JB. Erectile dysfunction is common among patients with gout. J Rheumatol. 2015;42(10):1893-7.

3. Chen YF, Lin HH, Lu CC, Hung CT, Lee MH, Hsu CY, Chung WS. Gout and a subsequent increased risk of erectile dysfunction in men aged 64 and under: a nationwide cohort study in Taiwan. J Rheumatol. 2015;42(10):1898-905.

4. Hsu CY, Lin CL, Kao CH. Gout is associated with organic and psychogenic erectile dysfunction. Eur J Intern Med. 2015;26(9):691-5.

5. Campion EW, Glynn RJ, DeLabry LO. Asymptomatic hyperuricemia. Risks and consequences in the Normative Aging Study. Am J Med. 1987;82(3):421-6.

6. Liddle J, Roddy E, Mallen CD, Hider SL, Prinjha S, Ziebland S, Richardson JC. Mapping patients' experiences from initial symptoms to gout diagnosis: a qualitative exploration. BMJ Open. 2015;5(9):e008323.

7. Kuo CF, Grainge MJ, Mallen C, Zhang W, Doherty M. Comorbidities in patients with gout prior to and following diagnosis: case-control study. Ann Rheum Dis. 2016;75(1):210-7.

8. Kuo CF, Grainge MJ, Mallen C, Zhang W, Doherty M. Rising burden of gout in the UK but continuing suboptimal management: a nationwide population study. Ann Rheum Dis. 2015;74(4):661-7.

9. Kuo CF, Grainge MJ, Mallen C, Zhang W, Doherty M. Effect of allopurinol on all-cause mortality in adults with incident gout: propensity score-matched landmark analysis. Rheumatology (Oxford, England). 2015;54(12):2145-50. 